\title{
Hybrid Purity Testing of Brassica rapa Using SSR Marker Technology
}

\section{$\mathrm{Li} \mathrm{Li}^{1}$ and Ling Liu}

Quality Supervision and Inspection and Testing Center of Vegetable and Seed of Beijing, Beijing 100097, China; Beijing Vegetable Research Center, Beijing Academy of Agricultural and Forestry Sciences, Beijing 100097, China; Key Laboratory of Horticultural Crops Biology and Germplasm Innovation, Ministry of Agriculture in North China, Beijing 100097, China; Key Laboratory of Urban Agriculture (North) of Ministry of Agriculture, Beijing 100097, China; and Beijing Jingyanyinong Technology Development Center, Beijing 100097, China

\section{Deshuang Zhang}

Beijing Vegetable Research Center, Beijing Academy of Agricultural and Forestry Sciences, Beijing 100097, China; Key Laboratory of Horticultural Crops Biology and Germplasm Innovation Ministry of Agriculture in North China, Beijing 100097, China; Key Laboratory of Urban Agriculture (North) of Ministry of Agriculture, Beijing 100097, China; and Beijing Jingyanyinong Technology Development Center, Beijing 100097, China

\section{Ping Wu}

Quality Supervision and Inspection and Testing Center of Vegetable and Seed of Beijing, Beijing 100097, China; Beijing Vegetable Research Center, Beijing Academy of Agricultural and Forestry Sciences, Beijing 100097, China; Key Laboratory of Horticultural Crops Biology and Germplasm Innovation Ministry of Agriculture in North China, Beijing 100097, China; Key Laboratory of Urban Agriculture (North) of Ministry of Agriculture, Beijing 100097, China; and Beijing Jingyanyinong Technology Development Center, Beijing 100097, China

\section{Fenglan Zhang}

Beijing Vegetable Research Center, Beijing Academy of Agricultural and Forestry Sciences, Beijing 100097, China; Key Laboratory of Horticultural Crops Biology and Germplasm Innovation Ministry of Agriculture in North China, Beijing 100097, China; Key Laboratory of Urban Agriculture (North) of Ministry of Agriculture, Beijing 100097, China; and Beijing Jingyanyinong Technology Development Center, Beijing 100097, China

\section{Xiulan Xu}

Quality Supervision and Inspection and Testing Center of Vegetable and Seed of Beijing, Beijing 100097, China; Beijing Vegetable Research Center, Beijing Academy of Agricultural and Forestry Sciences, Beijing 100097, China; Key Laboratory of Horticultural Crops Biology and Germplasm Innovation Ministry of Agriculture in North China, Beijing 100097, China; Key Laboratory of Urban Agriculture (North) of Ministry of Agriculture, Beijing 100097, China; and Beijing Jingyanyinong Technology Development Center, Beijing 100097, China

Additional index words. Brassica rapa ssp. pekinensis, Brassica rapa chinensis group, hybrid, SSR markers, purity identification, special strain

Abstract. Thirteen Chinese cabbage (Brassica rapa) hybrid cultivars and 26 parental inbred lines were used as experimental materials to screen for primers producing hybrid and parental complementary bands and for primers with high polymorphism information contents and low genotype frequencies. A total of 18 pairs of core primers were designed to identify the purity of Chinese cabbage. There was no significant difference in the purity percentage measured between different loci of the same strain. The fingerprint obtained by the amplification of each locus could be used to identify purity to obtain an authentic purity percentage. Curve mapping and significance analyses were conducted using the purity percentage of eight different seed samples and confirmed a sampling seed number of 96. The results of the purity test were verified by comparison with the grow-out test (GOT) using molecular markers. In conclusion, the simple sequence repeat (SSR) detection system could be used for the rapid identification of the purity of the tested Chinese cabbage hybrids.

Chinese cabbage (Brassica rapa) belongs to the Brassicaceae family and includes heading (Brassica rapa ssp. pekinensis) and nonheading (Brassica rapa L. chinensis group) subspecies (Ke, 2010). There are many excellent varieties of Chinese cabbage, making it a popular dish that can be served throughout the year.

Currently, most commercial cultivars of Chinese cabbage are hybrids. During the production of Chinese cabbage hybrid seeds, low temperatures, continuous overcast conditions, or rainy weather at the flowering stage will decrease the purity of the cultivar because of flowering asynchronism. These situations can result in irreparable economic losses to farmers as the generation of inbred seedlings will result in failure to meet the quality criterion of the seeds stipulated in contracts.

The conventional method for identifying the purity of Chinese cabbage hybrids is the GOT; however, this approach cannot easily discriminate off-type individuals at the seedling stage. In addition, because of the limited morphological traits of Chinese cabbage hybrids, one must wait until the maturation period to perform the GOT. Thus, because of the limited time span of the plant, it is difficult to identify the purity of most Chinese cabbage hybrid cultivars. Accordingly, a rapid, accurate, and effective method as well as regulations are necessary for identifying the purity of Chinese cabbage hybrids.

Biochemical technology has been applied to detect the purity of hybrids. For example, isoenzyme and protein electrophoretograms can distinguish Chinese cabbage hybrids from their parents (Huang and Zheng, 1994; Shi et al., 2014). However, the isoenzymes and proteins available are extremely limited. Furthermore, Chinese cabbage cultivars with close genetic relationships are difficult to screen. By contrast, SSR fingerprints have some obvious advantages, such as simplicity, clarity, and 
accuracy (Li and Zheng, 2009; Li et al., 2009; Shi et al., 2007; Suwabe et al., 2002). Indeed, SSR markers are gradually being applied for the identification of hybrid purity, including rice (Oryza sativa L.) (Sundaram et al., 2008), pigeon pea (Cicer microphyllum Royle ex Benth) (Saxena et al., 2010), sunflower (Helianthus annuus L.) (Pallavi et al., 2011), cabbage (Brassica oleracea L.) (Li et al., 2011; Liu et al., 2007), cauliflower (Brassica oleracea L. var. botrytis) (Zhao et al., 2012), melon (Pedicellus melo) (Li et al., 2015), and bulb onion (Allium cepa L.) (McCallum et al., 2008). Research has indicated that SSR markers are more reliable, practical, and efficient compared with the commonly used GOT method.

The most important factors in genetic purity determination using SSR markers are the number of core primers that are sufficient for distinguishing a cultivar, the number of individuals who need to be examined to estimate the purity percentage for a seed sample, and the number of SSR loci used to assess the purity of one hybrid sample in practical testing. However, very little is known regarding the cost effectiveness or labor intensity of this approach.

The aim of this study was to identify the purity of the 13 most commonly planted Chinese cabbage hybrids and to generate an economic and efficient method for identifying Chinese cabbage hybrid genetic purity using SSR molecular technology.

\section{Materials and Methods}

Materials. Seeds of the Chinese cabbage (Brassica rapa ssp. pekinensis and Brassica rapa L. chinensis) used in this study were obtained from the Chinese Cabbage Research Subject of the Beijing Vegetable Research Center of Beijing Academy of Agriculture and Forestry. The tested sample materials, including 13 hybrids and their parental inbred lines (a total of 39 samples), are listed in Table 1. Analyses were conducted in the Quality Supervision and Inspection and Testing Center of Vegetable and Seed of Beijing and the Seed Technique Molecular Detection Laboratory of the Vegetable Research Center

\footnotetext{
Received for publication 12 Apr. 2017. Accepted for publication 22 May 2017.

This research was supported by a Project of the Committee of Science and Technology of Beijing City (No. D131100000413001), the certification and testing and service capability promotion of the 2013-14 ISTA, the International Seed Testing Laboratory of Ascension, and the Beijing Finance Project (no item number) from 2009 to 2016. For cultivar testing for DNA fingerprint detection in the Beijing region, support was received from the National Science and Technology Support Program (No. 2011BAD35B07) from 2011 to 2015, the Beijing Academy of Agriculture and Forestry Finance Special Subject (KJCX20140111) from 2014 to 2016, and Beijing Academy of Agriculture and Forestry (KJCX20150202).

1Corresponding author. E-mail: 1ili@nercv.org.
}

of the Beijing Academy of Agriculture and Forestry Sciences in 2014.

Preparation of DNA. Five-day-old seedlings, $50-60 \mathrm{mg}$ of the small and tender leaves of mature plants, or the small and tender main leaves in the stem tip position of eugenic plants were collected and placed in 1.5-mL centrifuge tubes for DNA extraction. The method of extraction was based on a modified sodium dodecyl sulfate method (Li and Zheng, 2009).

Screening of primers for SSR markers. Sequences of 30 pairs of Brassica SSR core primers were screened (Choi et al., 2007; Dong et al., 2008; Li and Zheng, 2009; Li et al., 2009; Wei et al., 2012; Zhang and Li, 2013) and synthesized at SANGON Biotech Co., Ltd. (Shanghai, China) and SBS Genetech Co., Ltd. (Beijing, China). Only 18 pairs of primers (Table 2) were selected for use in the current experiments.

The polymerase chain reaction (PCR) mixture contained $0.4 \mu \mathrm{L} \quad 10 \mu \mathrm{mmol} \cdot \mathrm{L}^{-1}$ dNTPs, $2 \mu \mathrm{L} 10 \times$ PCR buffer, $0.15 \mu \mathrm{L}$ $5 \mathrm{U} \cdot \mu \mathrm{L}^{-1}$ Taq, $2 \mu \mathrm{L}$ 60-200 ng. $\mu \mathrm{L}^{-1}$ template DNA, $2 \mu \mathrm{L}$ forward primer, $2 \mu \mathrm{L}$ reverse primer, and water to a total volume of $20 \mu \mathrm{L}$. PCR was performed under the following conditions: $94{ }^{\circ} \mathrm{C}$ for $5 \mathrm{~min} ; 35$ cycles of $94{ }^{\circ} \mathrm{C}$ for $15 \mathrm{~s}, 55^{\circ} \mathrm{C}$ for $15 \mathrm{~s}$, and $72{ }^{\circ} \mathrm{C}$ for $30 \mathrm{~s} ; 72{ }^{\circ} \mathrm{C}$ for $4 \mathrm{~min}$; and $4{ }^{\circ} \mathrm{C}$ for storage. The amplification reaction was carried out using a PE9700 amplification apparatus from Gene Company Limited (Beijing, China). The amplified products were detected by electrophoresis through an ultra-highresolution agarose gel from AMRESCO Inc. (Solon, OH), with 3\% GoodView from SBS Genetech Co., Ltd. Imaging was performed using a Multimage gel-imaging apparatus produced by Alpha Innotech Company.

Data analysis.

$P$ [Hybrid purity percentage $(\%)]=$

Number of hybrid genotypes $\times 100 \%$

$\overline{\text { Number of genotypes of all individuals }}$

$t$-Test $1=(\mathrm{P} 1-\mathrm{P} 2) / \mathrm{STDEV} ; t$-Test $2=$ (P3 - P2)/STDEV

STDEV $($ standard deviation $)=S /(n-1)$

$S$ is the variance of the hybrid purity percentage for eight groups of individuals tested, and $n-1$ is the df.

$\mathrm{P} 1$ is the percentage of 96 individuals, $\mathrm{P} 2$ is the percentage of 192 individuals, and P3 is the percentage of 120 individuals.

$t$-Test $3=(\mathrm{P} 2 \mathrm{~s} 1-\mathrm{P} 2 \mathrm{~s} 2) /\left(\mathrm{DX}_{\mathrm{A}} / \mathrm{n} 1+\mathrm{DX}_{\mathrm{B}} / \mathrm{n} 2\right)^{1 / 2}$ $\mathrm{DX}_{\mathrm{A}}$ is the variance of one group for the percentage of purity measured at three loci of the same variety, and $\mathrm{DX}_{\mathrm{B}}$ is the variance of another group for the percentage of purity measured at three loci of the same variety.

$\mathrm{P} 2 \mathrm{~s} 1$ is the percentage of 192 individuals measured at one locus of the same variety, and P2s2 is the percentage of 192 individuals measured at another locus of the same variety.
The lowest genotype frequencies $=$ the number of specific genotype varieties/the number of all genotype varieties.

\section{Results}

Analysis of the SSR fingerprint spectrum of 13 Chinese cabbage hybrids and their parental inbred lines. Primers that produce heterozygous bands are good candidates for purity identification. Seedlings generated from hybrid seeds, as well as the seeds of each parent, were collected to confirm that BRU05680, Syau-m01, Syau-m05, cnu-m090a, and PBCGSSRBR24 are suitable primers for identifying Beijing new No. 3. The hybrids and their parents showed complementary band types or heterozygous band types. The inbred seeds, which were mixed into the hybrids, were clearly distinguished because the inbred genotype is one homozygous band. The actual genotype frequencies of these primers were also sufficiently low (Tables 1 and 2). Fito0528, Syau-m05, BRMS296, and BRMS-042-2 identified Xiaoza No. 60 and Xiaoza No. 61. However, some special strains with different band types were also present among Xiaoza No. 60 and Xiaoza No. 61. The primers selected for Beijing 68 were Fito0528, BRU05680, BRMS296, and CX273016, which were able to simultaneously detect the hybrid inbred seeds and heterotypic strains, such as special strains detected at the BRMS296 locus, as shown in Fig. 1.

The SSR core primers (30 pairs) for Chinese cabbage were applied to the 13 hybrid combinations. As illustrated in Table 1, more than two SSR alleles with complementary band types of the parents and hybrids were found for each hybrid combination. The primers used for the detection of Guoxia No. 1 were nia-m038a and Syau-m05. The primers cnu-m252a, cnu-m149a, P193, and BRMS296 were used to detect Jingyanheiye, and BRU07947, cnu-m149a, BRMS296, and niam038a were used for Naibai No. 1. Because of their complementary band types, the primers used to amplify the BRU07947 and enu-m149a loci were able to identify the special strains (Figs. 2 and 3). The primers used to detect Jingyankuaicai No. 4 included cnu-m252a, cnum149a, Syau-m05, PBCGSSRBR24, and BRMS-042-2 (Table 1). The parents and hybrids of Jingxia new No. 4 exhibited complementary band types when primers based on Syau-m01, Syau-m05, cnu-m252a, cnu-m149a, and cnu-m149a were applied. Jingguan No. 3 could be detected using BRU07947, cnum149a, P193, Syau-m05, BRMS296, and EJU3, which distinguished inbred seeds among the hybrids and identified specific strains in Jingguan No. 3 (Table 2; Fig. 4). In addition, seven, two, and three primer sets were found for the practical detection of hybrids $11 \mathrm{~N} 1,10 \mathrm{~N} 7$, and $11 \mathrm{~N} 5$, respectively (Table 1).

Identification of the purity of Chinese cabbage hybrids using SSR markers. It remained to be determined how many individuals should be tested to assess genetic purity and how many SSR loci would be 
Table 1. Samples of Chinese cabbage.

\begin{tabular}{|c|c|c|c|c|}
\hline$\overline{\text { No. }}$ & Name & Type & Taxonomy & Parental polymorphic SSR markers \\
\hline 3 & $\mathrm{P} 90$ & Inbred & A & \\
\hline 4 & Jingxia new No. 4 & Hybrid & A & Syau-m01, Syau-m05, cnu-m252a, cnu-m149a \\
\hline 6 & P84 & Inbred & A & \\
\hline 7 & Beijing xiaoza No. 60 & Hybrid & A & Fito0528, BRU05680, BRMS296, CX273016 \\
\hline 8 & $\mathrm{P} 4$ & Inbred & A & \\
\hline 11 & $\mathrm{P} 21$ & Inbred & A & \\
\hline 12 & P22 & Inbred & A & \\
\hline 13 & Beijing No. 68 & Hybrid & A & Fito0528, BRU05680, BRMS296, CX273016 \\
\hline 14 & P29 & Inbred & A & \\
\hline 15 & P30 & Inbred & A & \\
\hline 16 & Beijing new No. 3 & Hybrid & A & BRU05680, Syau-m05, cnu-m090a, Syau-m01, PBCGSSRBR24 \\
\hline 17 & P14 & Inbred & A & \\
\hline 23 & N13 & Inbred & B & \\
\hline 24 & $\mathrm{~N} 24$ & Inbred & B & \\
\hline 25 & $10 \mathrm{~N} 7$ & Hybrid & B & OL11H09, NA14G06 \\
\hline 26 & $\mathrm{~N} 21$ & Inbred & B & \\
\hline 27 & N29 & Inbred & B & \\
\hline 28 & $11 \mathrm{~N} 1$ & Hybrid & B & BRMS-042-2, OL11H09, EJU3, BRU07947, P193, BRMS296, Syau-m01 \\
\hline 29 & N38 & Inbred & B & \\
\hline 30 & N42 & Inbred & B & \\
\hline 31 & $11 \mathrm{~N} 5$ & Hybrid & B & Nia-m038a, CX273016, P193 \\
\hline 32 & N38 & Inbred & B & \\
\hline 33 & N43 & Inbred & B & \\
\hline 34 & Naibai No. 1 & Hybrid & B & BRU07947, cnu-m149a, BRMS296, nia-m038a \\
\hline 35 & N4 & Inbred & B & \\
\hline
\end{tabular}

A means Brassica rapa ssp. pekinensis of the heading type of Chinese cabbage. B means Brassica rapa L. chinensis group of the nonheading type of Chinese cabbage.

Table 2. Primer name, sequences, heterozygosity, and varieties distinguished and lowest genotype frequencies.

\begin{tabular}{|c|c|c|c|c|}
\hline $\begin{array}{l}\text { Primer } \\
\text { name }\end{array}$ & Forward primer sequence $\left(5^{\prime}-3^{\prime}\right)$ & Reverse primer sequence $\left(5^{\prime}-3^{\prime}\right)$ & $\begin{array}{l}\text { No. of varieties } \\
\text { distinguished }\end{array}$ & $\begin{array}{l}\text { Lowest genotype } \\
\text { frequencies }\end{array}$ \\
\hline BRU05680 & CATGTCGTCTTCTTTGCTG & CTTTTGCTTGAGCTTTGTGT & 2 & 0.059 \\
\hline BRU07947 & CATGTCGTCTTCTTTGCTG & CTTTTGCTTGAGCTTTGTGT & 3 & 0.036 \\
\hline cnu-m149a & GGAAGCCTCTGTGCGAAAAA & TGCCGACGATTTGATAGAGGA & 6 & 0.018 \\
\hline P193 & TCAAGATCCTCCACTTCT & ACGGACTGTATAGCGACT & 7 & 0.020 \\
\hline Syau-m01 & GTGAGTTAAGCACCGACAG & ACAGAGGACCATCAGCAT & 3 & 0.034 \\
\hline EJU3 & ССТСТTТTAАTТCAAACAAGAAATCA & TTCGGACAATGGCAGTGATA & 4 & 0.018 \\
\hline CX273016 & AGA GAG ATC GAG ATC CAC AA & GGC GAG AAA GTT AGT TGC TA & 2 & 0.021 \\
\hline PBCGSSRBR24 & CAG ACA GAC TTT GGT TGG AG & ATA GGT CAG GAG AGG TGA AGA & 4 & 0.034 \\
\hline nia-m038a & GGGCCAAGTTACATGGAAAA & GAAGGAGGATGAGAGCCGTT & 5 & 0.018 \\
\hline NA14G06 & AAACGGCTTGCATTGTTCTC & GGCTTGCTTGATCCAGTCTC & 3 & 0.111 \\
\hline OL11H09 & СССТТТТССССТТСТАТTGG & GTGCGACTTGGAATTTCTCC & 3 & 0.021 \\
\hline cnu-m090a & GCAAAGATCGGCGAAGAAGA & TGCAGACACATTCGAACAAACA & 2 & 0.018 \\
\hline
\end{tabular}

needed for the purity testing of one hybrid. To address these issues, three loci of Beijing new No. 3-1, Jingguan No. 3, and Beijing No. 68 were selected, and 192 seeds were examined (Table 3). Significance analysis between two groups regarding the purity percentage measured at the three loci of the same variety is shown in Table 4 , with no significant difference $\left(t\right.$-TEST3 $\left.<t_{0.05(14)}=2.145\right)$. Two loci were selected for Guoxia No. 1, Jingyanheiye, Naibai No. 1, 11N1, 10N7, Jingyankuaicai No. 4, Beijing new No. 3-2, and Beijing Xiaoza No. 61. Significance analyses of the measured percentages of purity at the two loci of the same cultivars are illustrated in Table 4. As the $t$-TEST3 result was smaller than $t_{0.05(14)}=2.145$, the difference was not significant. Thus, one locus was able to distinguish both inbred seeds and the special strains among the hybrids.

One locus with a complementary band type was selected for Beijing Xiaoza No. 60 , Jingxia new No. 4, and Beijing new No. 3-3 (Table 3). Curve analysis of the measured data for 192 seeds in the eight experimental groups of all 13 samples is shown in Fig. 5A and $\mathrm{B}$. The purity percentage of a given 


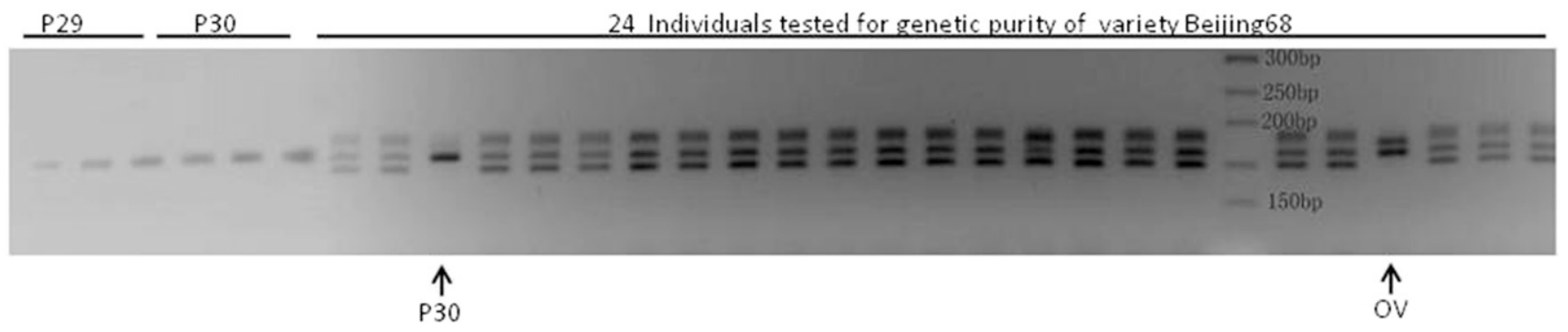

Fig. 1. Primer pair for BRMS296 used to screen for the hybrid Beijing 68. P29 and P30 are the parents, and OV is one other cultivar mixed in Beijing 68. P30 is mixed with the hybrid. The bands with size labeled are DNA molecular ladder.

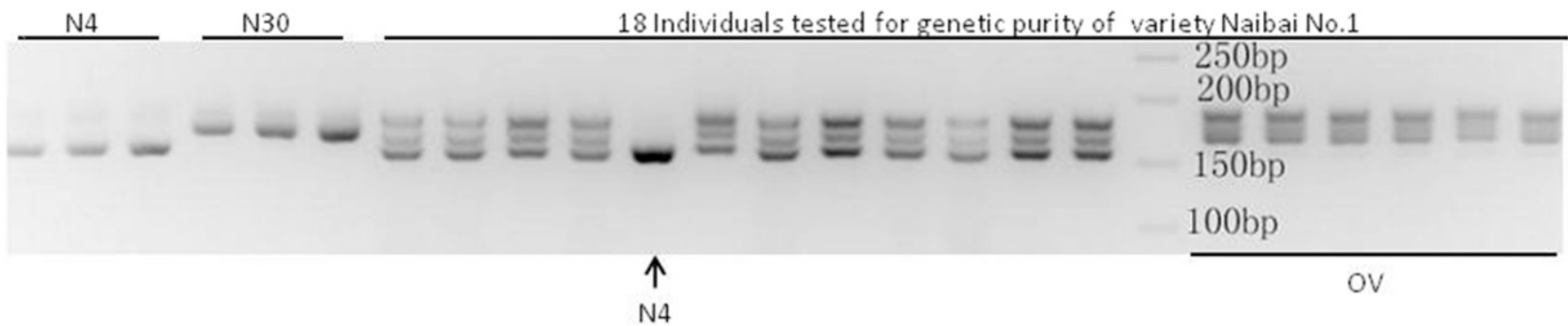

Fig. 2. Primer pair for BRU07947 used to screen for the hybrid Naibai No. 1. N4 and N30 are the parents, and OV contains a mixture of other cultivars present in Naibai No. 1. N4 is mixed with the hybrids. The bands with size labeled are DNA molecular ladder.

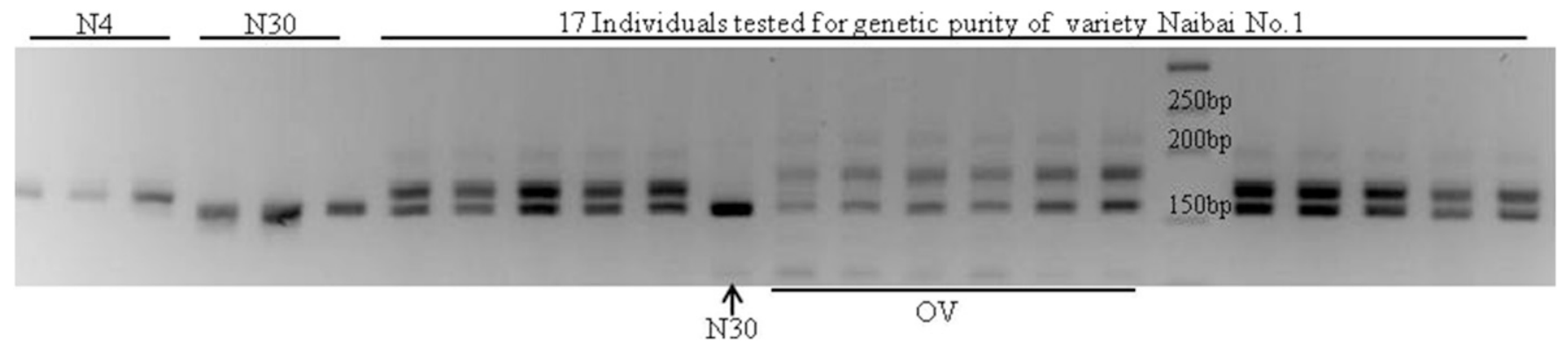

Fig. 3. Primer pair for cnu-m149a used to screen for the hybrid Naibai No. 1. N4 and N30 are the parents, and lanes marked "OV" show other cultivars mixed in Naibai No. 1. N30 is mixed with the hybrids. The bands with size labeled are DNA molecular ladder.

hybrid tended to stabilize as the number of seeds examined increased. Regarding the data obtained for the four groups of 96 seeds (the determined sampling number), we observed a notable fluctuation range for the purity percentage. In addition, the purity percentage tended to be similar for the testing of 96 seeds of the four groups (Fig. 5A and B). This suggested that the probability of error for the detected purity percentage was significant when the number of seeds examined was less than 96. By comparison, the possibility of error for the detected purity percentage was considered to be minimal if the number of seeds evaluated was greater than 96. These results of the significance analysis for the purity percentage of each hybrid and their final values are shown in Table 4. $t$-Test1 refers to the significance analysis for the percentage of 96 individuals vs. the percentage of 192 individuals, and $t$-Test 2 refers to the significance analysis for the percentage of 120 individuals vs. the percentage of 192 individuals. $P<t_{0.05(7)}=2.365$ for $t$-Test1 and $t$-Test2, with no significant difference. Therefore, a random sampling of 96 seeds was deemed suitable for detection.

The Chinese Cabbage Research Group of the Beijing Vegetable Research Center sent the seed samples for hybrid purity testing using SSR markers. Simultaneously, breeders identified the hybrid purity using the GOT. The resulting purity percentages determined by two methods are listed in Table 5 . The purity percentage for each cultivar tested using SSR makers for 96 individuals was used. These results deviated $0.1 \%$ to $4.6 \%$ between SSR fingerprint detection and the GOT. For seedlings 5-7 d old, including weak growing seedlings, DNA was extracted and analyzed for hybrid purity percentage by applying SSR fingerprint detection. Meanwhile, in the GOT, only the parents could be distinguished from F1 hybrids during the mature period. Some weak individuals died because of unsuitable growing conditions. Normally, inbred individuals die easily because of their weak growing tendency. Therefore, purity percentages determined by SSR methods were higher than those determined by the GOT by no more than $5.0 \%$.

The difference in the hybrid purity percentages detected by SSR fingerprint detection and the GOT was statistically analyzed. The $t$-Test of the purity percentages determined by the two methods produced a value of 0.656 , which was far less than $t_{0.05(7)}=1.895$. The difference was not significant. It was concluded that the purity percentage measured by SSR markers could replace the purity percentage identified in the GOT.

Specificity of primers and identification of special strains. The selection of low genotype frequencies was important for identifying special strains among the hybrids and involved specific primer-targeted loci. In the present study, the genotype frequency of the selected primer-targeted loci was low, and inconsistent band types for special strains were observed (Table 3 ). The banding patterns of the special strains during the seedling 


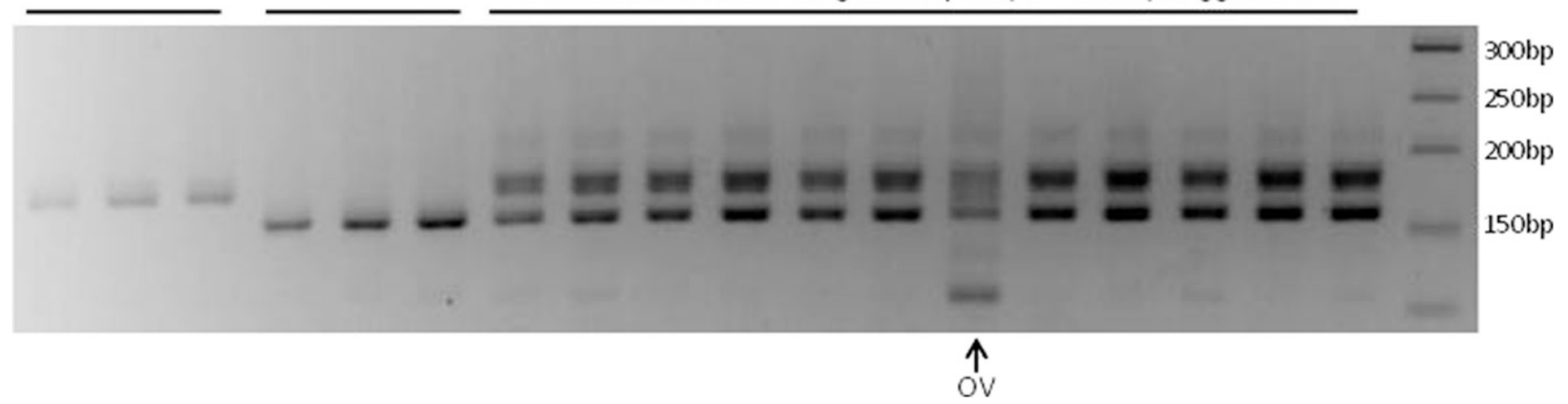

Fig. 4. Primer pair for BRU07947 used to screen for the hybrid Jingguan No. 3. N13 and N24 are the parents, and OV is one other cultivar mixed in Jingguan No. 3. $M$ is the DNA molecular ladder.

Table 3. Hybrid purity percentage for each variety determined by different simple sequence repeat (SSR) primers and different numbers of individuals.

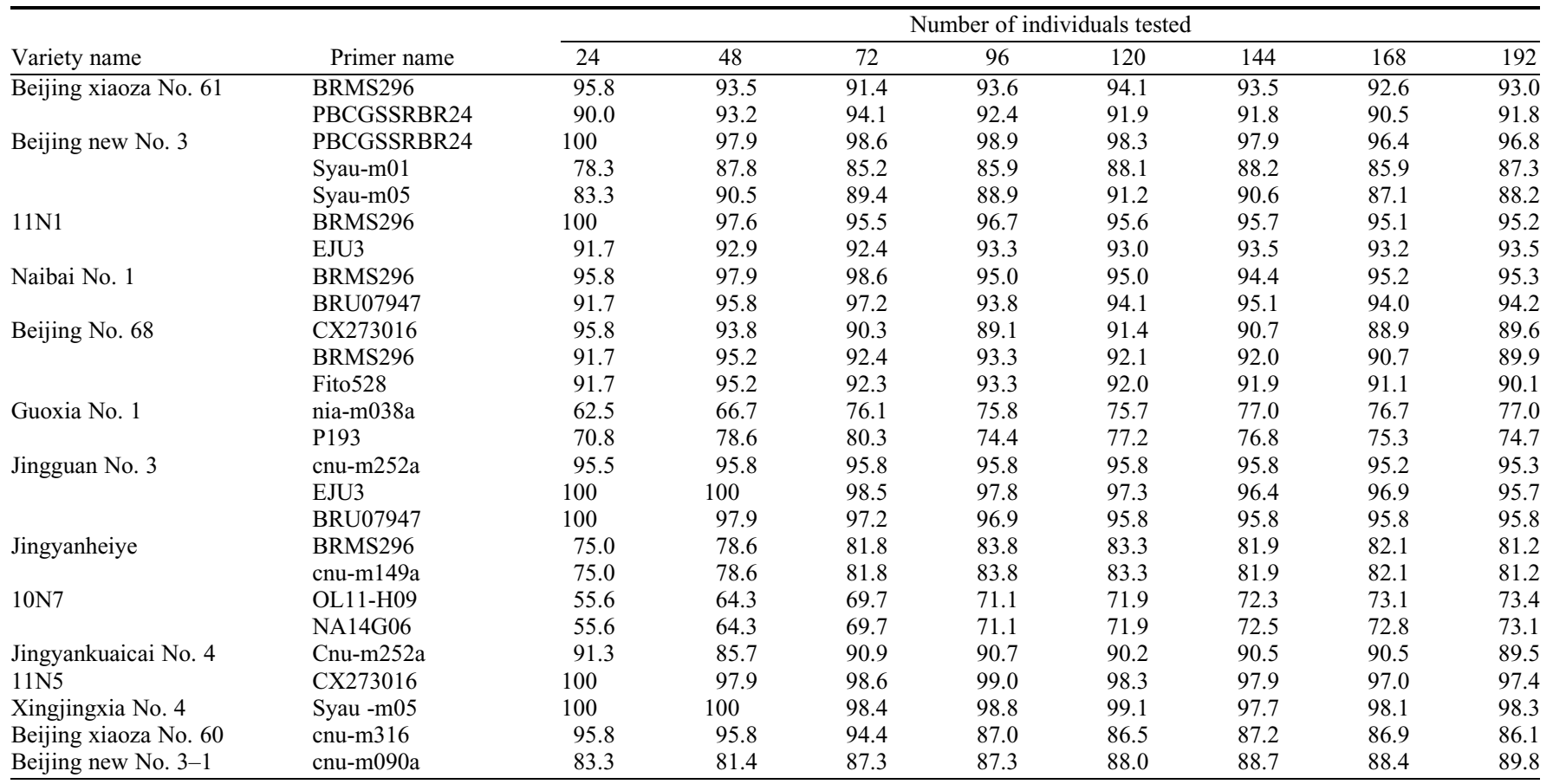

period were compared at the SSR loci of Jingguan No. 3 and Beijing No. 68 (Figs. 1 and 2).

\section{Discussion}

Hybrid purity and identification of authenticity are important for both the quality and supervision of seeds. In addition to many other advantages, the high polymorphism of SSR markers makes this method of molecular identification convenient for analyses with high reproducibility, stability, and efficiency. Indeed, this approach is increasingly being applied for the marker-assisted selection of plants. SSR markers have gradually been used to identify the purity of cultivars, such as in maize (Zea mays L.) (Jia, 2008), watermelon (Citrullus landaus Thunb.) (Zhou et al., 2012), rape (Brassica napus L.) (Dong et al., 2008), cabbage (Li et al., 2011), Chinese cabbage (Suwabe et al., 2006; Wang et al., 2008; Wei et al., 2012), and other important crops. SSR markers have been used for gene mapping (Suwabe et al., 2006) and to establish genetic linkage maps (Choi et al., 2007; Li et al., 2012; Wang et al., 2011; Yu et al., 2009; Yuan et al., 2010). Our laboratory began to develop expressed sequence tag-SSR markers in 2007; these markers have been applied for the construction of the core germplasm of Chinese cabbage (Li and Zheng, 2009; Li et al., 2009), as well as for the establishment of a DNA fingerprint database (Zhang and Li, 2013). Core primers for SSR markers have also been used to detect the purity of Chinese cabbage hybrids, implementing a molecular method to assess the authenticity of varieties. To facilitate the breeding and selling of seeds under supervised conditions, the main Chinese cabbage varieties planted in northern China were used as the experimental material in this study. In the process of testing the purity of the samples and during the authenticity experiments, we continuously communicated with breeders and analyzed the experimental material to provide a solid basis for the use of SSR molecular markers in evaluating the technical specifications for Chinese cabbage purity.

With regard to purity detection using the DNA fingerprinting technique, the first priority was the screening of primers. It is highly important to obtain a set of core primer pairs for genetic purity testing of varieties. The 18 screened pairs of SSR primers in the present research were based on the core primers produced for the construction of the fingerprint database of Chinese cabbage by the Seed Technique Molecular Detection Laboratory ( $\mathrm{Li}$ and Zheng, 2009; Li et al., 2009; Shi et al., 2007). As they exhibited the required characteristics of core primers for parental complementary band types of the hybrids during the identification of Chinese cabbage purity, each pair of primers was able to identify parental inbred seeds of three to seven strains among all 13 varieties. However, 


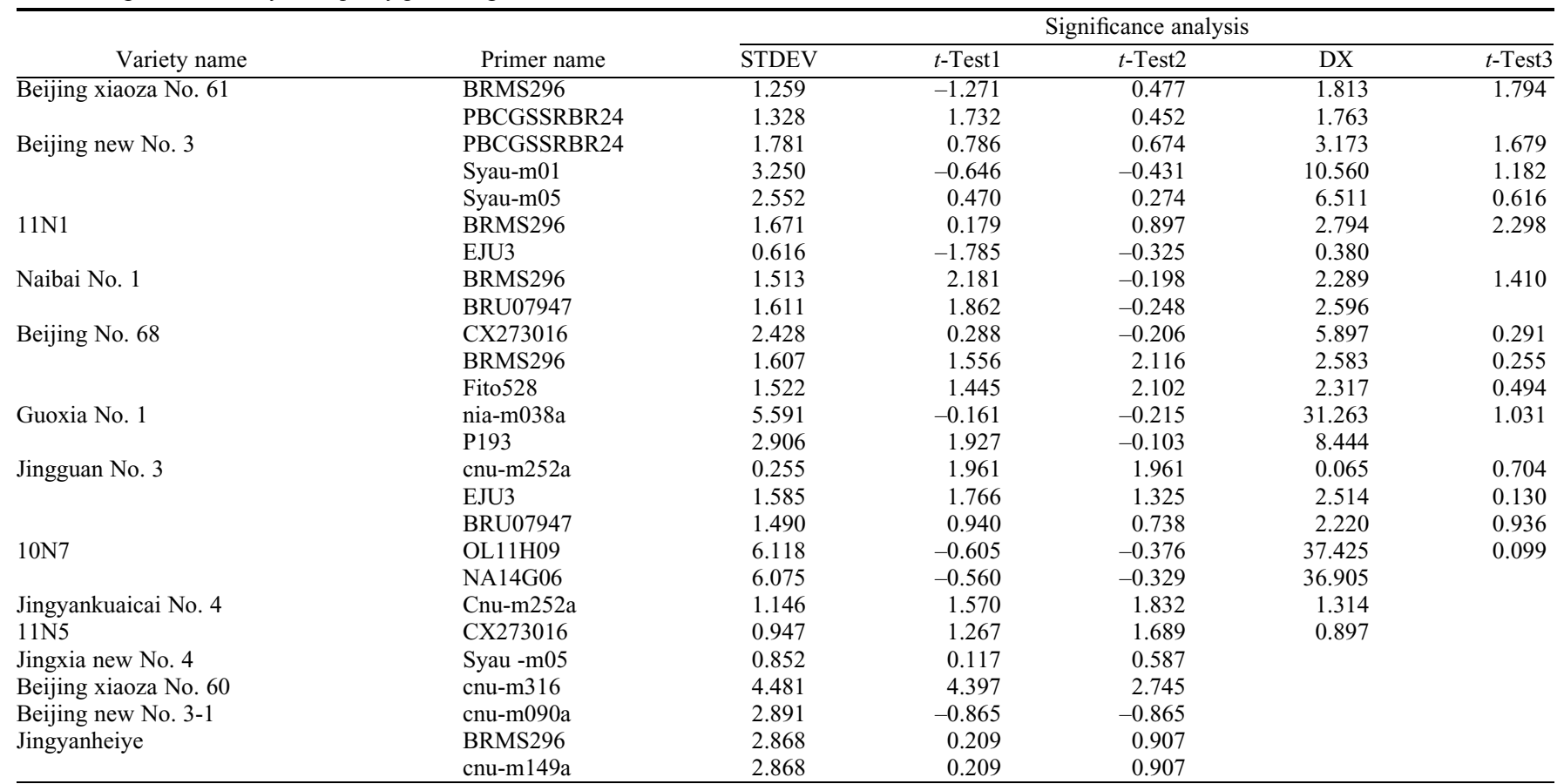

$t$-Test 1 is the significance analysis of the percentage of 96 individuals vs. the percentage of 192 individuals.

$t$-Test 2 is the significance analysis of the percentage of 120 individuals vs. the percentage of 192 individuals.

$t$-Test 3 is the significance analysis between the two groups for the purity percentage measured at three loci of the same variety.

STDEV $=$ standard deviation

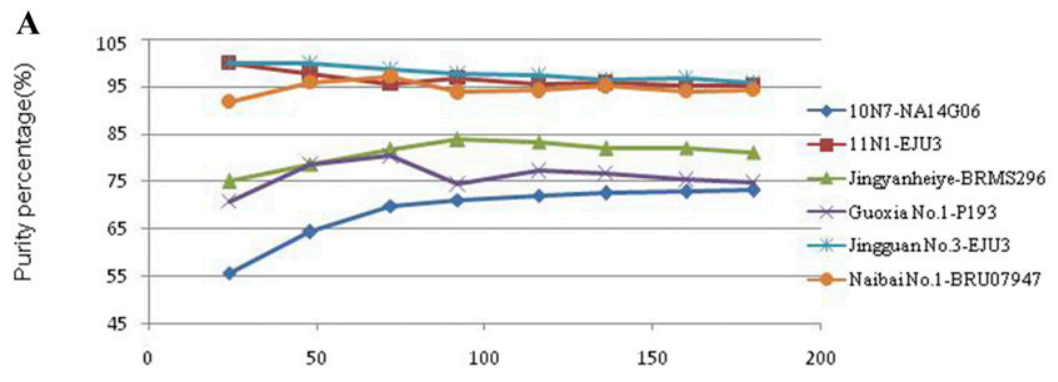

Numbers of individuals tested

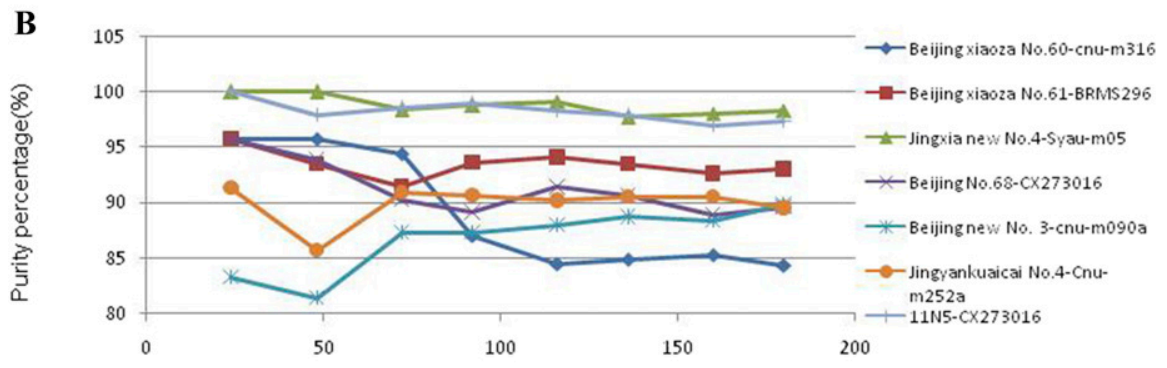

Numbers of individuals tested

Fig. 5. (A) Curve analysis of the purity percentage for seven samples. (B) Curve analysis of the purity percentage for six samples. Regarding the data obtained from the four groups of 96 seeds, the fluctuation range of the purity percentage was obvious, and the purity percentage tended to be similar.

the genotype frequency of the primer Syau-m05 was slightly higher, at 0.111 . Nonetheless, this primer was selected based on the parent and hybrids of the six hybrid combinations with parental complementary band types for this primer and because it could detect parental inbred seedlings in the hybrid. The genotype frequencies of the other 16 pairs of primers were relatively low. During the detection of the mixed parental inbred seedlings in the hybrid, this primer was also used to identify other cultivars. That is, it was specific for a particular cultivar and therefore could be used in authenticity testing of a specific variety because it could distinguish the special strains of other cultivars mixed in the hybrid while detecting parental inbred seedlings.

The advantages of molecular markers for identifying the purity of Chinese cabbage are that they are economical and efficient and save both labor and time.

In previous studies, three to five SSR loci and 400 seeds of a rice hybrid were used for purity assessment (Sundaram et al., 2008), and two SSR loci and 379 and 91 seeds from seed lots of hybrids of pigeon pea were used to identify purity (Saxena et al., 2010). In addition, two SSR loci and 200 individual hybrid seeds were used for cauliflower (Zhao et al., 2012), whereas one SSR locus and 100 individual hybrids were used for melon ( $\mathrm{Li}$ et al., 2015). We wondered whether the results would be more accurate when more individuals and loci were tested, even though it is more time consuming to test more individuals and more loci for the identification of one hybrid sample. The issues in testing can be contradictory. For example, some customers require that 300 individuals be tested to assess hybrid purity, although they want the results as quickly as possible. Therefore, suitable sampling seed and locus numbers need to be defined. Here, we address the question pertaining to the number of seeds required to obtain the purity percentage, and the methods used were found to be accurate and reliable. Curve mapping and significance analysis were performed on the purity percentage for eight different strains, confirming that 96 is a suitable number of seeds sampled.

Two pairs of primers were screened for each cultivar or line. Based on significance analysis 
Table 5. Hybrid purity percentage as determined by simple sequence repeat (SSR) markers and by the grow-out test.

\begin{tabular}{|c|c|c|c|c|c|c|c|c|}
\hline & Beijing xiaoza No. 61 & $11 \mathrm{~N} 1$ & Xinjingxia No. 4 & Naibai No. 1 & Guoxia No. 1 & Jingguan No. 3 & $11 \mathrm{~N} 5$ & Jingyankuaicai No. 4 \\
\hline SSR fingerprint (\%) & 93.0 & 95.0 & 98.8 & 94.4 & 75.1 & 96.8 & 99.0 & 90.7 \\
\hline Classical methods (\%) & 93.9 & 96.8 & 98.9 & 95.3 & 76.9 & 100 & 100 & 95.3 \\
\hline
\end{tabular}

of the measured purity percentage of the same strain at different sites, no significant differences in this value between the various loci were found (Table 4). Thus, for a real and reliable purity percentage, purity identification could be conducted using amplification fingerprints at each site. Changes in season and environmental temperatures influence the humidity in the laboratory, which can affect the speed at which temperature is changed in the apparatus used for amplification. It should be noted that this problem was encountered, the reason for which was commonly agreed on, and a summary of the experiments was communicated to the related technical personnel of the company that synthesized the primers. Another problem was the synthesis of primers in different batches, with inevitable changes in band types during the experiments, as more than two primers could identify the purity of each strain. During seed harvest, one primer set could be rapidly replaced by another primer set for purity measurement. When a large number of samples need to be identified quickly, this detection method with improved efficiency can meet the demands of seed sales for seed producers in a timely manner and can meet the urgent needs of vegetable producers.

\section{Literature Cited}

Choi, S.R., G.R. Teakle, P. Plaha, J.H. Kim, C.J. Allender, E. Beynon, Z.Y. Piao, P. Soengas, T.H. Han, G.J. King, G.C. Barker, P. Hand, D.J. Lydiate, J. Batley, D. Edwards, D.H. Koo, J.W. Bang, B.S. Park, and Y.P. Lim. 2007. The reference genetic linkage map for the multinational Brassica rapa genome sequencing project. Theor. Appl. Genet. 115:777-792.

Dong, Y.Y., M.N. Yu, X.B. Li, P.F. Xu, H.R. Cui, and M.L. Zhang. 2008. Diversity analysis of Brassica napus by EST-SSR and RAPD markers. J. Nucl. Agr. Sci. 22:611-616.

Huang, W.P. and X.Y. Zheng. 1994. Peroxidase isozyme analysis of $\mathrm{F}_{1}$ hybrid and parent seedling in Chinese cabbage (Brassica rapa L. ssp. pekinensis). Seed 71:13-15.

Jia, X-H. 2008. SSR molecular maps of hybrid maize varieties. China Agr. Press, Beijing, China.

Ke, G-L. 2010. Chinese cabbage breeding. China Agr. Press, Beijing, China.

Li, L., W.M. He, L.P. Ma, P.Y. Liu, H.M. Xu, J.B. Xu, and X.Y. Zheng. 2009. Construction
Chinese cabbage (Brassica rapa L.) core collection and its EST-SSR fingerprint database by EST-SSR molecular markers. Genomics Appl. Biol. 28:76-88.

Li, L., Y.C. Jian, L.P. Ma, X.Q. Li, and Y.H. Ding. 2011. Cabbage (Brassica oleracea) hybrid purity quotient test by EST-SSR molecular makers. Genomics Appl. Biol. 30:1161-1164.

Li, L., W.Q. Zhang, L. Liu, P. Wu, and L.P. Ma. 2015. Application of SSR markers in melon hybrid purity test and variety specificity identification. Mol. Plant Breed. 13:2522-2530.

Li, L. and X.Y. Zheng. 2009. The development of multiplex EST-SSR markers to identification Chinese cabbage (Brassica campestris L. chinensis (L.) Makino and Brassica campestris $\mathrm{L}$. pekinensis (Lour.) Olsson) cultivar. Acta Hort. Sin. 36:1627-1634.

Li, Y.F., Zh.Y. Liu, Y.Sh. Wang, N. Yang, X.F. Xin, Sh. Yang, and H. Feng. 2012. Identification of quantitative trait loci for yellow inner leaves in Chinese cabbage (Brassica rapa $\mathrm{L}$. ssp. pekinensis) based on SSR and SRAP markers. Sci. Hort. 133:10-17.

Liu, G., L. Liu, Y. Gong, Y. Wang, F. Yu, H. Shen, and W. Gui. 2007. Seed genetic purity testing of F1 hybrid cabbage (Brassica oleracea var. capitata) with molecular marker analysis. Seed Sci. Technol. 35:477-486.

McCallum, J., S. Thomson, P.J. Meeghan, and K. Fernand. 2008. Genetic diversity analysis and single-nucleotide polymorphism marker development in cultivated bulb onion based on expressed sequence tag-simple sequence repeat markers. J. Amer. Soc. Hort. Sci. 133:810-818.

Pallavi, H.M., R. Gowda, Y.G. Shadakshari, K. Bhanuprakash, and K. Vishwanath. 2011. Identification of SSR markers for hybridity and seed genetic purity testing in sunflower (Helianthus annuus L.). Seed Sci. Technol. 39:259-264.

Saxena, R.K., K. Saxena, and R.K. Varshney. 2010. Application of SSR markers for molecular characterization of hybrid parents and purity assessment of ICPH 2438 hybrid of pigeonpea (Cajanus cajan L. Millspaugh). Mol. Breed. 26:371-380.

Shi, L., L. Li, and X.Y. Zheng. 2007. Optimization of SSR-PCR system for identification in Chinese cabbage (Brassica campestris L. ssp. pekinensis). Mol. Plant Breed. 5:110-116.

Shi, P.H., Z.G. Liu, Y.H. Zhang, W.C. Sun, D.J. Kong, M.H. Lu, and N.N. Yang. 2014. Analysis on antioxidant enzyme activities and peroxidase isozymes in 23 rape cultivars. Acta Agr. Boreali-occidentlis Sin. 23:113-119.

Sundaram, R.M., B. Naveenkumar, S.K. Biradar, S.M. Balachandran, B. Mishra, M. IlyasAhmed,
B.C. Viraktamath, M.S. Ramesha, and N.P. Sarma. 2008. Identification of informative SSR markers capable of distinguishing hybrid rice parental lines and their utilization in seed purity assessment. Euphytica 163:215-224.

Suwabe, K., H. Iketani, T. Nunome, T. Kage, and M. Hirai. 2002. Isolation and characterization of microsatellites in brassica rapa L. Theor. Appl. Genet. 104:1092-1098.

Suwabe, K., H. Tsukazaki, H. Iketani, K. Hatakeyama, M. Kondo, M. Fujimura, T. Nunome, H. Fukuoka, M. Hirai, and S. Matsumoto. 2006. SSR-based comparative genomics between Brassica rapa and Arabidopsis thaliana: The genetic origin of clubroot resistance. Genetics 173:309-319.

Wang, X.Y., Sh.C. Yu, F.L. Zhang, Y.J. Yu, X.Y. Zhao, and D.Sh. Zhang. 2008. SSR fingerprinting and genetic distinctness of Pak-choi (Brassica rapa $\mathrm{L}$. ssp. chinensis Makino). Acta Agr. Boreali Sin. 23:97-103.

Wang, Y., S.L. Sun, B. Liu, H. Wang, J. Deng, Y.C. Liao, Q. Wang, F. Cheng, X.W. Wang, and J. Wu. 2011. A sequence-based genetic linkage map as a reference for Brassica rapa pseudo chromosome assembly. BMC Genomics 12:239.

Wei, L.Y., C.X. Zhao, J.N. Li, J.N. Li, W. Qian, and D.H. Fu. 2012. Screening of core simple sequence repeat primer pairs and establishment of a multiplex polymerase chain reaction system for brassica genomes A and C. Plant Breed. 131:457-459.

Yu, S.C., F.L. Zhang, R.B. Yu, Y.M. Zou, J.N. Qi, X.Y. Zhao, Y.J. Yu, D.S. Zhang, and L. Li. 2009. Genetic mapping and localization of a major QTL for seedling resistance to downy mildew in Chinese cabbage (Brassica rapa ssp. pekinensis). Mol. Breed. 23:573-590.

Yuan, Y.X., X.W. Zhang, R.F. Sun, X.W. Wang, H. Zhang, W.S. Jiang, Q.J. Yao, and J.F. Geng. 2010. Construction of a genetic linkage of Chinese map anchoring to chromosomes cabbage. Acta Agr. Boreali-Sin.. 25:80-86.

Zhang, W. and L. Li. 2013. Construction of SSR fingerprint database of Chinese cabbage varieties (Brassica campestris L. ssp. pekinensis). Mol. Plant Breed. 11:843-857.

Zhao, Z., H. Gu, X. Sheng, H. Yu, J. Wang, and J. Cao. 2012. Genetic purity testing of loosecurd cauliflower hybrids using SSR markers and grow out test. Seed Sci. Technol. 40:209-214.

Zhou, X.D., F.Ch. Wang, G.L. Zhou, X.F. Zhu, M. Lin, L.L. Chen, and H.B. Wang. 2012. Testing of genetic purity of watermelon $\mathrm{F}_{1}$ varieties by SSR markers. China Cucurb. Veg. 25:13-16. 